\title{
EFEITOS DA ADUBAÇÃO NITROGENADA E COM MICRONUTRIENTES NA QUALIDADE DE SEMENTES DO FEIJOEIRO CULTIVAR IAC-CARIOCA ${ }^{(1)}$
}

\author{
EDMILSON JOSÉ AMBROSANO ${ }^{(2,5)}$, GLÁUCIA MARIA BOVI AMBROSANO ${ }^{(2)}$, ELAINE BAHIA \\ WUTKE ${ }^{(3)}$, EDUARDO ANTONIO BULISANI (3), ANTONIO LÚCIO MELLO MARTINS (4) \\ \& LUIS CLÁUDIO PATERNO SILVEIRA ${ }^{(3)}$
}

\begin{abstract}
RESUMO
Foram realizados ensaios de laboratório com amostras de sementes obtidas de dois experimentos em campo, no município de Pindorama, de maio a agosto, em 1992 e 1993, visando estudar o efeito do nitrogênio e de micronutrientes, aplicados sob várias formas, na qualidade das sementes do feijoeiro cultivar IAC-Carioca. Tomaram-se amostras de sementes de cada tratamento aplicado em campo, acondicionando-as em sacos de papel e conservando-as em câmara seca com temperatura de $15^{\circ} \mathrm{C}$ e umidade relativa do ar de $40 \%$, sendo efetuados testes de germinação imediatamente após a colheita e após 12 e 24 meses. Os resultados foram analisados e as médias, comparadas pelo teste de Duncan: tanto o fornecimento diferencial de $\mathrm{N}$ como o de micronutrientes não afetaram a qualidade fisiológica das sementes, medida por meio da percentagem de germinação e de plântulas anormais, não se observando acúmulo ou déficit de elementos nutrientes nas sementes do feijoeiro.
\end{abstract}

Termos de indexação: feijão, Phaseolus vulgaris L., nitrogênio, micronutrientes, irrigação, qualidade de semente.

\footnotetext{
(1) Recebido para publicação em 10 de julho de 1998 e aceito em 25 de março de 1999.

(2) Bioestatística, Departamento de Odontologia Social, FOP-UNICAMP, Avenida Barão de Limeira, 901, 13414-018 Piracicaba (SP).

(3) Centro de Plantas Graníferas, Instituto Agronômico (IAC), Caixa Postal 28, 13001-970 Campinas (SP).

(4) Estação Experimental de Agronomia de Pindorama, IAC, Caixa Postal 24, 15830-000 Pindorama (SP).

${ }^{(5)}$ Com bolsa de produtividade em pesquisa do CNPq.
} 


\title{
ABSTRACT \\ THE EFFECT OF NITROGEN AND MICRONUTRIENT ON SEED QUALITY OF DRY BEANS
}

\begin{abstract}
Germination tests of cv. IAC Carioca were conducted with seeds from two field experiments in 1992 and 1993. Nitrogen and micronutrients, were applied in the yield various forms and the quality of seeds was assessed. Seed samples were packed in paper bags and maintained in a cold dry chamber at a temperature of $15^{\circ} \mathrm{C}$ and $40 \%$ relative humidity. Germination tests were conducted immediately after harvest and 12 and 24 months of storage. The data were analyzed by comparing the average by the Duncan test. The results showed that the different sources of $\mathrm{N}$ and micronutrients did not affect the quality of seeds and that there was no excess or deficiency of nutrients in the dry beans seeds.
\end{abstract}

Index terms: dry beans, Phaseolus vulgaris L., nitrogen, micronutrients, irrigation, seed quality.

A qualidade de sementes pode ser expressa pela interação de quatro componentes: genético, físico, fisiológico e sanitário. No presente estudo o interesse é o componente fisiológico, que se refere ao potencial de longevidade da semente e a sua capacidade para gerar planta perfeita e vigorosa. A qualidade fisiológica diz respeito a atributos intrínsecos à semente, os quais determinam a sua capacidade de germinar e emergir rapidamente para que a cultura se estabeleça e produza plantas vigorosas, mesmo quando as condições no campo não sejam as mais adequadas. Essa capacidade fisiológica de desempenho da semente é modificada continuamente com o tempo, sendo tais modificações conhecidas como processo de deterioração: este é irreversível, culminando com a morte da semente.

O teste de germinação é o método mais usado e reconhecido para medição da qualidade fisiológica da semente, embora tenha as suas limitações, pois as condições a que a semente é submetida para germinar são próximas às mais adequadas (Bragantini, 1996).

Segundo Vieira et al. (1993b), o componente fisiológico é influenciado pelo ambiente em que as sementes se formam e pelas condições de colheita, de secagem, de beneficiamento e de armazenamento.

O presente estudo foi realizado em laboratório com amostras de sementes obtidas de ensaios realiza- dos em campo durante dois anos consecutivos, em Pindorama, com o objetivo de avaliar o efeito da adubação nitrogenada e com micronutrientes, aplicadas via solo ou foliar, em doses únicas ou parceladas, na qualidade fisiológica da semente produzida.

\section{Material e Métodos}

O ensaio foi realizado em laboratório com amostras de sementes obtidas de dois ensaios efetuados durante dois anos consecutivos, no período de maio a agosto, no município paulista de Pindorama (1992 e 1993), ambos em um solo podzolizado vermelho-amarelo, utilizando-se o cultivar de feijão IACCarioca e irrigação por aspersão.

As sementes e os dados de produção de grãos foram obtidos por Ambrosano et al. (1996a, b). As sementes foram acondicionadas em sacos de papel e conservadas em câmara seca com temperatura de $15^{\circ} \mathrm{C}$ e umidade relativa do ar de $40 \%$. Avaliaram-se os efeitos da adubação nitrogenada e com micronutrientes mediante testes de germinação, imediatamente após a colheita e 12 e 24 meses depois, os quais levaram em conta a percentagem de germinação e o número de plântulas anormais. Avaliaram-se ainda, as concentrações de nutrientes nas sementes. 
O efeito do nitrogênio na qualidade das sementes foi estudado em sementes obtidas dos seguintes tratamentos aplicados ao feijoeiro: testemunha sem adição de nitrogênio e com adubação básica; testemunha sem nitrogênio e sem adubação básica; aplicação de dose total de $\mathrm{N}\left(60 \mathrm{~kg} \cdot \mathrm{ha}^{-1}\right.$ de $\left.\mathrm{N}\right)$, no plantio, aos 15 dias após germinação (DAG), aos 25 DAG, 1/ 2 da dose no plantio e $1 / 2$ aos $15,1 / 2$ da dose no plantio e $1 / 2$ aos 25 DAG, $1 / 2$ aos 15 e $1 / 2$ aos 25 DAG,e de aplicações foliares aos 15, 25, 35 e 45 DAG, de uma solução contendo uréia a $5 \%$ e $10 \%$, sendo um tratamento com $1 / 2$ da dose no plantio e $1 / 2$ com pulverizações com $5 \%$ de uréia e outro tratamento com toda dose $\left(60 \mathrm{~kg} \cdot \mathrm{ha}^{-1}\right.$ de $\left.\mathrm{N}\right)$ em pulverizações com $10 \%$ de uréia. O delineamento experimental foi o de blocos ao acaso, com 10 tratamentos e 5 repetições.

$\mathrm{O}$ efeito de micronutrientes na qualidade das sementes foi estudado em sementes obtidas dos se- guintes tratamentos aplicados ao feijoeiro: testemunha sem adição de micronutrientes e com a adubação básica; testemunha sem micronutrientes e sem a adubação básica; aplicação de $20 \mathrm{~kg} \cdot \mathrm{ha}^{-1}$ de Borogram (8\% B); 20 kg.ha-1 de Zincogram (20\% Zn); 30 kg.ha ${ }^{1}$ de FTE-BR-12 (9\% de Zn; $1,8 \%$ de B; $0,8 \%$ de Cu; 3,0\% de Fe; 2,0\% de Mn e 0,1\% de Mo); 60 kg.ha-1 de FTE-BR-12; e de aplicações foliares, aos 15, 25 e 35 dias após emergência, de uma solução diluída contendo N, P, K (1,0: 0,5: 1,4 kg.ha- ${ }^{-1}$ de N, $\mathrm{P}_{2} \mathrm{O}_{5}, \mathrm{~K}_{2} \mathrm{O}$ respectivamente) e outra que, além de $\mathrm{N}, \mathrm{P}, \mathrm{K}$, incluía uma mistura de micronutrientes contendo boro, molibdênio e zinco, na forma de 340, 68 e 1.700 g.ha${ }^{1}$ de ácido bórico, molibdato de sódio e sulfato de zinco respectivamente. $\mathrm{O}$ delineamento experimental foi de blocos casualizados com cinco repetições.

Foram feitas análises da variância e as médias dos tratamentos, comparadas pelo teste de Duncan.

Quadro1. Efeito da adubação nitrogenada sobre os teores de macro- e de micronutrientes em sementes de feijoeiro ('IAC-Carioca') cultivado no inverno em Pindorama (1992/1993)

\begin{tabular}{|c|c|c|c|c|c|c|c|c|c|c|c|c|c|c|c|c|}
\hline \multicolumn{17}{|c|}{ Adubação } \\
\hline \multicolumn{3}{|c|}{ Solo } & \multicolumn{4}{|c|}{ Foliar } & \multicolumn{5}{|c|}{1992} & \multicolumn{5}{|c|}{1993} \\
\hline 0 & 15 & 25 & 15 & 25 & 35 & $45 \mathrm{DAG}^{(1)}$ & $\mathrm{N}$ & $\mathrm{P}$ & K & B & $\mathrm{Zn}$ & $\mathrm{N}$ & $\mathrm{P}$ & K & B & $\mathrm{Zn}$ \\
\hline \multicolumn{3}{|c|}{ kg.ha- ${ }^{-1}$ de $\mathrm{N}$} & \multicolumn{4}{|c|}{ Pulverização c/ uréia (\%) } & \multicolumn{3}{|c|}{ g. $\mathrm{kg}^{-1}$} & \multicolumn{2}{|c|}{$\mathrm{mg} \cdot \mathrm{kg}^{-1}$} & \multicolumn{3}{|c|}{ - g. $\mathrm{kg}^{-1}$} & \multicolumn{2}{|c|}{ mg.kg ${ }^{-1}$} \\
\hline 60 & - & - & - & - & - & - & 26,2 & 4,7 & 11,5 & 11 & 43,2 & 32,0 & 5,2 & 10,5 & 12 & 40,0 \\
\hline 一 & 60 & - & - & - & - & - & 24,6 & 4,3 & 10,5 & 10 & 36,8 & 25,2 & 4,3 & 10,5 & 15 & 44,2 \\
\hline - & - & 60 & - & - & - & - & 28,6 & 4,4 & 11,0 & 9 & 41,5 & 28,0 & 4,4 & 12,5 & 13 & 40,4 \\
\hline 30 & 30 & - & - & - & - & - & 26,8 & 4,4 & 11,0 & 8 & 39,9 & 28,6 & 4,7 & 12,0 & 14 & 45,1 \\
\hline 30 & - & 30 & - & - & - & - & 25,9 & 4,6 & 12,0 & 10 & 40,5 & 28,2 & 4,6 & 11,5 & 12 & 43,1 \\
\hline - & 30 & 30 & - & - & - & - & 25,1 & 4,4 & 11,0 & 10 & 38,2 & 27,0 & 4,7 & 11,5 & 13 & 40,8 \\
\hline 30 & - & - & 5 & 5 & 5 & 5 & 25,4 & 4,8 & 11,5 & 10 & 37,6 & 26,8 & 4,8 & 10,5 & 14 & 38,5 \\
\hline - & - & - & 10 & 10 & 10 & 10 & 24,2 & 5,0 & 12,0 & 14 & 38,1 & 26,4 & 4,8 & 11,5 & 15 & 41,1 \\
\hline \multicolumn{7}{|c|}{ Test. com adubação básica sem N .......... } & 26,2 & 4,8 & 10,0 & 12 & 37,3 & 27,0 & 4,7 & 11,5 & 15 & 38,4 \\
\hline \multicolumn{7}{|c|}{ Test. sem adubação básica sem N ........... } & 24,2 & 4,7 & 11,0 & 13 & 37,3 & 27,9 & 4,9 & 12,0 & 16 & 39,0 \\
\hline
\end{tabular}

${ }^{(1)}$ Dias após germinação. 


\section{Resultados e Discussão}

Observam-se nos Quadros 1 e 4 os teores de macro- e de micronutrientes nas sementes de feijoeiro cultivar IAC-Carioca, para os ensaios com aplicação de nitrogênio e micronutrientes, respectivamente, nos dois anos de observação. Nota-se que os teores de nutrientes variaram pouco, indicando que a semente tende a absorver nutrientes em proporções definidas.

Ambrosano et al. (1998) encontraram um acumulo de Ni em sementes de feijoeiro produzidas em solo em que foram aplicados $47 \mathrm{mg}$ de Ni por $\mathrm{kg}$ de solo. Os teores de Ni na semente do feijoeiro passaram de $0,68 \mathrm{mg} \cdot \mathrm{kg}^{-1}$ para $102,91 \mathrm{mg} \cdot \mathrm{kg}^{-1}$ nos tratamentos testemunha e aplicação de $47 \mathrm{mg}$ de Ni por $\mathrm{kg}$ de solo, indicando a acumulação pela semente.

Observa-se, no presente estudo, que os elementos minerais não foram aplicados em excesso como o efetuado por Ambrosano et al. (1998), e, sim, em doses recomendadas para garantir um equilíbrio nutricional. Esse fato pode explicar, em parte, a falta de respostas em termos de acumulo de nutrientes nas sementes.
Nota-se nos Quadros 2 e 5 - percentagem de germinação e plântulas anormais, dos ensaios de nitrogênio e de micronutrientes respectivamente, nas três épocas de amostragem - que a percentagem de germinação decresceu com o tempo de amostragem, enquanto a de plântulas anormais aumentou, confirmando, assim, a queda na qualidade da semente com o decorrer do tempo.

Na primeira determinação, as sementes estão dentro dos padrões de qualidade, que sugere uma germinação mínima de $80 \%$ para o feijoeiro. Segundo Vieira et al. (1993a), após um ano de armazenamento de sementes de feijoeiro, em condições adequadas, a percentagem de germinação decresceu para valores abaixo do mínimo permitido para a cultura.

Segundo Vieira et al. (1993b), além do ambiente em que as sementes são produzidas e as condições de armazenamento, é de grande importância, também, o cultivar analisado, pois, dependendo do material, esse decréscimo da germinação pode ser mais acentuado: a queda da germinação do 'Milionário' se deu aos 183 dias da colheita em comparação com 486 dias do 'Fortuna'.

Quadro 2. Efeito da adubação nitrogenada na percentagem de germinação e de plântulas anormais em diferentes tempos de amostragem no feijoeiro 'IAC - Carioca'

\begin{tabular}{|c|c|c|c|c|}
\hline \multirow{2}{*}{$\begin{array}{c}\text { Tempo } \\
\text { de } \\
\text { amostragem }\end{array}$} & \multicolumn{2}{|c|}{1992} & \multicolumn{2}{|c|}{1993} \\
\hline & Germinação & Anormais & Germinação & Anormais \\
\hline & \multicolumn{2}{|c|}{$-\%$} & 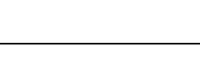 & 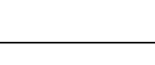 \\
\hline $0 \ldots \ldots \ldots \ldots . .$. & 85,8 a & $7,7 \mathrm{c}$ & $96,1 \mathrm{a}$ & $2,9 \mathrm{~b}$ \\
\hline 1 .................... & $61,1 \mathrm{~b}$ & $29,5 \mathrm{~b}$ & $53,2 \mathrm{~b}$ & $42,7 \mathrm{a}$ \\
\hline $2 \ldots \ldots \ldots \ldots \ldots$ & $35,1 \mathrm{c}$ & 53,9 a & $49,5 \mathrm{c}$ & $43,8 \mathrm{a}$ \\
\hline $\mathrm{CV} \% \ldots \ldots \ldots$ & 7,2 & 13,4 & 2,9 & 5,2 \\
\hline
\end{tabular}

Médias seguidas de letras distintas na vertical diferem entre si pelo teste de Duncan $(\mathrm{P}<0,05)$. 
Quadro 3. Efeito da adubação nitrogenada na percentagem média de germinação e de plântulas anormais no feijoeiro 'IAC - Carioca'

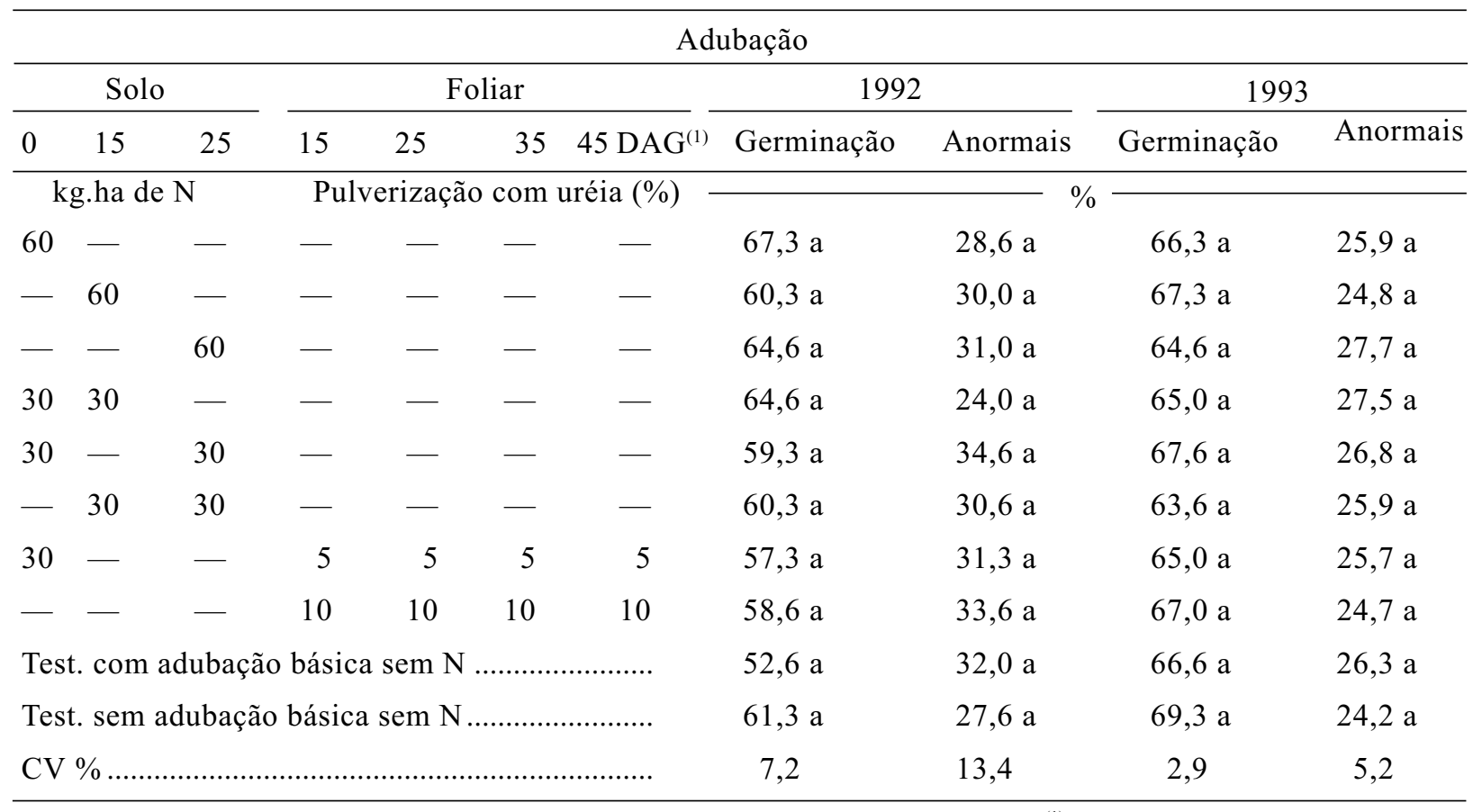

Médias seguidas de letras distintas na vertical diferem entre si pelo teste de Duncan $(\mathrm{P}<0,05) .{ }^{(1)}$ Dias após germinação.

Quadro 4. Efeito da adubação com micronutrientes sobre os teores de macro- e de micronutrientes em sementes do feijoeiro 'IAC-Carioca' cultivado no inverno

\begin{tabular}{|c|c|c|c|c|c|c|c|c|c|c|c|c|c|}
\hline \multicolumn{14}{|c|}{ Adubação } \\
\hline \multirow{2}{*}{ Solo ${ }^{(1)}$} & \multicolumn{3}{|c|}{ Foliar } & \multicolumn{5}{|c|}{1992} & \multicolumn{5}{|c|}{1993} \\
\hline & 15 & 25 & $3545 \mathrm{DAG}^{(2)}$ & $\mathrm{N}$ & $\mathrm{P}$ & K & B & $\mathrm{Zn}$ & $\mathrm{N}$ & $\mathrm{P}$ & $\mathrm{K}$ & B & $\mathrm{Zn}$ \\
\hline & \multicolumn{3}{|c|}{ pulverização c/ micro } & \multicolumn{3}{|c|}{$\mathrm{g} \cdot \mathrm{kg}^{-1}$} & \multicolumn{2}{|c|}{$\mathrm{mg} \cdot \mathrm{kg}^{-1}$} & \multicolumn{3}{|c|}{$\mathrm{g} \cdot \mathrm{kg}^{-1}$} & \multicolumn{2}{|c|}{$\mathrm{mg} \cdot \mathrm{kg}^{-1}$} \\
\hline Borogram & & $\mathrm{kg} \cdot \mathrm{h}$ & ${ }^{-1}$ no plantio) & 23,4 & 4,3 & 10,5 & 16 & 35,0 & 29,8 & 5,2 & 11,0 & 15 & 34,8 \\
\hline Zincogram & $(20$ & $\mathrm{kg} \cdot \mathrm{h}$ & ${ }^{-1}$ no plantio) & 25,0 & 4,1 & 10,5 & 10 & 36,7 & 31,6 & 5,7 & 12,5 & 11 & 41,6 \\
\hline FTE-BR-12 & $2(30$ & $\mathrm{kg} \cdot \mathrm{h}$ & $1^{-1}$ no plantio) & 28,9 & 4,2 & 11,0 & 14 & 33,6 & 30,5 & 5,4 & 12,5 & 13 & 36,8 \\
\hline FTE-BR-12 & $2(60$ & kg.he & $i^{-1}$ no plantio) & 27,7 & 4,0 & 11,5 & 16 & 33,3 & 30,7 & 5,6 & 12,0 & 15 & 39,1 \\
\hline NPK pulv. & 15 & 25 & $3545 \mathrm{DAG}^{(2)}$ & 26,0 & 5,0 & 11,0 & 10 & 34,9 & 27,2 & 4,7 & 12,5 & 13 & 40,9 \\
\hline \multicolumn{14}{|l|}{ NPK pulv. } \\
\hline \multicolumn{14}{|l|}{ + micro- } \\
\hline nutrientes & 15 & 25 & $3545 \mathrm{DAG}^{(2)}$ & 27,0 & 4,6 & 11,0 & 12 & 32,9 & 31,2 & 4,5 & 12,0 & 13 & 41,3 \\
\hline \multicolumn{4}{|c|}{ Test. com adubação básica sem micro } & 23,6 & 4,7 & 10,0 & 13 & 33,6 & 33,3 & 6,1 & 11,0 & 11 & 40,2 \\
\hline \multicolumn{4}{|c|}{ Test. sem adubação básica sem micro } & 26,5 & 5,3 & 10,5 & 13 & 35,5 & 28,0 & 4,6 & 11,5 & 14 & 39,9 \\
\hline
\end{tabular}


Quadro 5. Efeito da adubação com micronutrientes na percentagem de germinação e de plântulas anormais no feijoeiro 'IAC - Carioca' em diferentes tempos de amostragem

\begin{tabular}{|c|c|c|c|c|}
\hline \multirow{2}{*}{$\begin{array}{c}\text { Tempo } \\
\text { de } \\
\text { amostragem }\end{array}$} & \multicolumn{2}{|c|}{1992} & \multicolumn{2}{|c|}{1993} \\
\hline & Germinação & Anormais & Germinação & Anormais \\
\hline Anos & 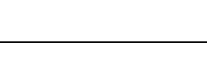 & & $x^{2}$ & \\
\hline $0 \ldots \ldots \ldots \ldots \ldots$ & $88 \mathrm{a}$ & $7 \mathrm{c}$ & 99 a & $0 \mathrm{~b}$ \\
\hline 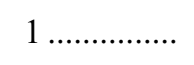 & $60 \mathrm{~b}$ & $30 \mathrm{~b}$ & $62 \mathrm{~b}$ & $35 \mathrm{a}$ \\
\hline $2 \ldots \ldots \ldots \ldots \ldots$ & $44 \mathrm{c}$ & $46 \mathrm{a}$ & $56 \mathrm{~b}$ & $39 a$ \\
\hline $\mathrm{CV} \% \ldots \ldots$. & 10,4 & 10,4 & 10,0 & 24,7 \\
\hline
\end{tabular}

Médias seguidas de letras distintas, na coluna, diferem entre si pelo teste de Duncan $(\mathrm{P}<0,05)$.

Quadro 6. Efeito da adubação com micronutrientes na percentagem média de germinação e de plântulas anormais do feijoeiro 'IAC - Carioca'

\begin{tabular}{|c|c|c|c|c|c|c|c|c|}
\hline \multicolumn{9}{|c|}{ Adubação } \\
\hline \multicolumn{5}{|c|}{ Foliar } & \multicolumn{2}{|c|}{1992} & \multicolumn{2}{|c|}{1993} \\
\hline & 15 & 25 & 35 & $45 \mathrm{DAG}^{(2)}$ & Germinação & Anormais & Germinação & Anormais \\
\hline kg.ha-1 de $\mathrm{N}$ & \multicolumn{4}{|c|}{ pulverização c/ micro } & \multicolumn{4}{|c|}{$-\%$} \\
\hline Borogram & \multicolumn{4}{|c|}{ (20 kg.ha- ${ }^{-1}$ no plantio) } & $68 \mathrm{a}$ & $25 \mathrm{a}$ & $72 \mathrm{a}$ & $27 \mathrm{a}$ \\
\hline Zincogram & \multicolumn{4}{|c|}{ (20 kg.ha-1 no plantio) } & $58 \mathrm{a}$ & $31 \mathrm{a}$ & $74 \mathrm{a}$ & $18 \mathrm{a}$ \\
\hline FTE-BR-12 & \multicolumn{4}{|c|}{ (30 kg.ha-1 no plantio) } & $61 \mathrm{a}$ & $29 \mathrm{a}$ & $77 \mathrm{a}$ & $22 \mathrm{a}$ \\
\hline FTE-BR-12 & \multicolumn{4}{|c|}{ (60 kg.ha-1 no plantio) } & $62 \mathrm{a}$ & $30 \mathrm{a}$ & $68 \mathrm{a}$ & $29 \mathrm{a}$ \\
\hline NPK pulv. & 15 & 25 & 35 & $45 \mathrm{DAG}^{(2)}$ & $71 \mathrm{a}$ & $25 \mathrm{a}$ & $75 \mathrm{a}$ & $25 \mathrm{a}$ \\
\hline \multicolumn{9}{|l|}{ NPK pulv. } \\
\hline \multicolumn{9}{|l|}{ + micro- } \\
\hline nutrientes & 15 & 25 & 35 & $45 \mathrm{DAG}^{(2)}$ & $61 \mathrm{a}$ & $26 \mathrm{a}$ & $73 \mathrm{a}$ & $24 \mathrm{a}$ \\
\hline \multicolumn{5}{|c|}{ Test. com adubação básica sem micronutrientes } & $67 \mathrm{a}$ & $28 \mathrm{a}$ & $70 \mathrm{a}$ & $27 \mathrm{a}$ \\
\hline \multicolumn{5}{|c|}{ Test. sem adubação básica sem micronutrientes } & $63 \mathrm{a}$ & $26 \mathrm{a}$ & $70 \mathrm{a}$ & $28 \mathrm{a}$ \\
\hline \multicolumn{5}{|l|}{$\mathrm{CV} \%$} & 10,4 & 10,4 & 10,0 & 24,7 \\
\hline
\end{tabular}

Médias seguidas de letras distintas, na coluna, diferem entre si pelo teste de Duncan $(\mathrm{P}<0,05)$.

(1) Adubação na semeadura. ${ }^{(2)}$ Dias após germinação. 
Observa-se - Quadros 3 e 6 - que os tratamentos não influenciaram a percentagem de germinação e de plântulas anormais, tanto nos ensaios de aplicação de nitrogênio como nos de micronutrientes, não tendo estes alterado a qualidade das sementes determinada pelo teste de germinação.

Segundo Bragantini (1996), a capacidade fisiológica de desempenho das sementes é modificada continuamente com o tempo, sendo tais modificações conhecidas como processo de deterioração. Tal processo, comum a todos os organismos vivos, culmina com a morte da semente.

\section{Conclusões}

Nem o fornecimento diferencial de $\mathrm{N}$ nem o de micronutrientes afetaram a qualidade fisiológica das sementes, medida através da percentagem de germinação e de plântulas anormais, não tendo sido observado acúmulo ou déficit de elementos nutrientes nas sementes do feijoeiro.

\section{Agradecimentos}

Os autores agradecem aos Engenheiros Agrônomos Helvécio Della Coleta Filho e José Eduardo Lelis, o apoio, e às técnicas de Apoio à Pesquisa Científica e Tecnológica, Ocimara Aparecida Alves, Virgínia Maria Barbosa Villar, Luzia de Fátima da Silva e Valéria Garcia, o preparo das amostras de sementes.

\section{Referências bibliográficas}

AMBROSANO, E.J; BERTON, R.S.; ABREU, C.A de \& Pires, A.M.M. Validation of annual loading limit stipulated by USEPA for Ni on Oxisol cropped with common beans: In: WORLD CONGRESS OF SOIL SCIENCE, 16., Montpellier, 1998. Summaries. Montpellier, ISSS/AISS/ IBG/SICS, 1998. v.2, p.470

AMBROSANO, E.J.; WUTKE, E.B.; AMBROSANO, G.M.B.; BULISANI, E.A.; BORTOLETTO, N.; MARTINS, A.L.M.; PEREIRA, J.C.V.N.A. \& DE SORDI, G. Resposta da aplicação de micronutrientes no cultivo de feijão irrigado no inverno. Scientia Agricola, Piracicaba, 53(2/3):273-279, 1996 a.

AMBROSANO, E.J.; WUTKE, E.B.; AMBROSANO, G.M.B.; BULISANI, E.A.; BORTOLETTO, N.; MARTINS, A.L.M.; PEREIRA, J.C.V.N.A. \& DE SORDI, G. Efeito do nitrogênio no cultivo de feijão irrigado no inverno. Scientia Agricola, Piracicaba, 53(2/3):338-342, 1996 b.

BRAGANTINI, C. Produção de Sementes. In: ARAUJO, R.S.; RAVA, C.A.; STONE, L.F. \& ZIMMERMANN, M.J., eds. Cultura do feijoeiro comum no Brasil. POTAFOS, Piracicaba, 1996. p. 639-667.

VIEIRA, R.F.; FARIA, M.N.; RAMOS, J.A. de O.; VIEIRA, C. \& DONZELES, S.M.L. Poder germinativo de sementes de algumas espécies de Vigna e Phaseolus durante o armazenamento. In: REUNIÃO NACIONAL DE PESQUISA DE FEIJÃO, 4., LONDRINA, 1993. Resumos. Londrina, IAPAR, 1993a. 222p.

VIEIRA, R.F.; VIEIRA, C. \& RAMOS, J.A.de O. Produção de sementes de feijão. Viçosa, EPAMIG/EMBRAPA,1993b. $131 \mathrm{p}$. 